\title{
Normal form of particle motion under the influence of an ac dipole
}

\author{
R. Tomás \\ CERN, Geneva, Switzerland \\ (Received 8 April 2002; published 28 May 2002)
}

\begin{abstract}
ac dipoles in accelerators are used to excite coherent betatron oscillations at a drive frequency close to the tune. These beam oscillations may last arbitrarily long and, in principle, there is no significant emittance growth if the ac dipole is adiabatically turned on and off. Therefore the ac dipole seems to be an adequate tool for nonlinear diagnostics provided the particle motion is well described in the presence of the ac dipole and nonlinearities. Normal forms and Lie algebra are powerful tools to study the nonlinear content of an accelerator lattice. In this article a way to obtain the normal form of the Hamiltonian of an accelerator with an ac dipole is described. The particle motion to first order in the nonlinearities is derived using Lie algebra techniques. The dependence of the Hamiltonian terms on the longitudinal coordinate is studied showing that they vary differently depending on the ac dipole parameters. The relation is given between the lines of the Fourier spectrum of the turn-by-turn motion and the Hamiltonian terms.
\end{abstract}

DOI: 10.1103/PhysRevSTAB.5.054001

PACS numbers: 41.85.-p, 29.27.-a

\section{LINEAR MOTION WITH AN AC DIPOLE}

The simplified Hamiltonian that describes the linear motion of a particle in an accelerator with an ac dipole is given by

$$
H_{0}\left(x, p_{x}, s, t\right)=\frac{1}{2} p_{x}^{2}+\frac{1}{2} K_{x}(s) x^{2}-\delta(s, t) x,
$$

where $x$ and $p_{x}$ are the transverse canonical coordinates, $s$ is the longitudinal coordinate, $K_{x}(s)$ is the focusing strength, and $\delta(s, t)$ is the time-dependent kick of the ac dipole placed at the location $s_{D}$ given by the expression

$$
\delta(s, t)=\frac{B L}{\left(B_{0} \rho\right)} \delta_{\text {Dirac }}\left(s-s_{D}\right) \cos \left(2 \pi Q_{D} t+\psi_{0}\right),
$$

where $B L$ is the integrated field amplitude, $\left(B_{0} \rho\right)$ is the rigidity, $Q_{D}$ and $\psi_{0}$ are the tune and initial phase of the ac dipole, and $\delta_{\text {Dirac }}\left(s-s_{D}\right)$ is the Dirac delta function. Note that throughout this article the unperturbed linear machine is assumed to be free of transverse coupling to simplify the expressions. A generalization to include linear coupling requires an additional decoupling transformation throughout the formalism [1]. The exact single-turn map corresponding to the Hamiltonian of Eq. (1) at the location just before the ac kick can be found in [2]. Using the Courant-Snyder variables $\left(\hat{x}, \hat{p}_{x}\right)$ this solution is written as a function of the turn number $T$ as

$$
\begin{aligned}
\hat{x}(T)-i \hat{p}_{x}(T)= & \sqrt{2 J} e^{i\left(2 \pi Q_{x} T+\phi_{x 0}\right)} \\
& +\delta_{-} e^{i 2 \pi Q_{D} T}-\delta_{+} e^{-i 2 \pi Q_{D} T},
\end{aligned}
$$

where $J$ and $\phi_{x 0}$ are the linear invariant and the initial phase given by the initial conditions and $\delta_{-}$and $\delta_{+}$are defined as

$$
\delta_{ \pm}=\sqrt{\beta_{D}} \frac{B L}{\left(B_{0} \rho\right)} \frac{e^{ \pm i\left(\pi Q_{ \pm}-\psi_{0}\right)}}{4 \sin \left(\pi Q_{ \pm}\right)},
$$

where $\beta_{D}$ is the betatron function at the dipole and $Q_{ \pm}=$ $Q_{D} \pm Q_{x}$. Notice that close to the resonance $Q_{D}=Q_{x}$ the quantity $\delta_{-}$is much larger than $\delta_{+}$.

A general solution for the particle motion at any location of the ring can be computed following the same steps as in [2]. The equivalent expression of Eq. (3) at the longitudinal location $s$ is given by

$$
\begin{aligned}
\hat{x}(T)-i \hat{p}_{x}(T)= & \sqrt{2 J} e^{i\left(2 \pi Q_{x} T+\phi_{x 0}\right)} \\
& +e^{-i \phi_{D}}\left(\delta_{-} e^{i 2 \pi Q_{D} T}-\delta_{+} e^{-i 2 \pi Q_{D} T}\right),
\end{aligned}
$$

where $\phi_{D}$ is the phase advance from the starting location $s$ to the ac dipole. $J$ and $\phi_{x 0}$ are again given by the initial conditions. Notice that $\phi_{D}$ has a discontinuity at the ac dipole since right before the ac dipole $\phi_{D}$ is zero and right after it $\phi_{D}$ is equal to $2 \pi Q_{x}$.

\section{THE TIME-INDEPENDENT LINEAR ONE-TURN MAP}

In general, an explicitly time-dependent Hamiltonian can be transformed to a time-independent Hamiltonian by introducing an extra degree of freedom (this is equivalent to extend the phase space [3]). The Hamiltonian $H\left(x, p_{x}, t\right)$ generates the same equations of motion for $x$ and $p_{x}$ as the new Hamiltonian $\bar{H}\left(x, p_{x}, \tau, p_{\tau}\right)$, defined by

$$
\bar{H}\left(x, p_{x}, \tau, p_{\tau}\right)=H\left(x, p_{x}, \tau\right)+p_{\tau},
$$

since the solution of the canonical equations for $\tau$ is $\tau(t)=t$.

Applying this transformation to the time-dependent Hamiltonian of Eq. (1) a new Hamiltonian independent of time with 1 extra degree of freedom is obtained. The one-turn operator acting on $\tau$ is defined to be $R_{\tau}$, and its action over $\tau$ is

(C) 2002 The American Physical Society

054001-1 


$$
R_{\tau} \tau=\tau+1
$$

The total linear one-turn map is the direct product of the one-turn map of the betatron motion with the operator
$R_{\tau}$, this is expressed as $R_{x} \otimes R_{\tau}$, where $R_{x}$ is defined as $R_{x} \phi_{x}=\phi_{x}+2 \pi Q_{x}$. The turn-by-turn evolution of the particle at the location of the ac dipole is reexpressed as follows:

$$
\hat{x}(T)-i \hat{p}_{x}(T)=\left(R_{x} \otimes R_{\tau}\right)^{T}\left[\sqrt{2 J} e^{i\left(\phi_{x}+\phi_{x 0}\right)}+\delta_{-} e^{i 2 \pi Q_{D} \tau}-\delta_{+} e^{-i 2 \pi Q_{D} \tau}\right]
$$

The cross denoting the direct product will be omitted in the following.

\section{THE NONLINEAR ONE-TURN MAP}

Since the explicit time dependence of the Hamiltonian has been removed, the one-turn map including nonlinear kicks can be constructed as in [4]. All nonlinearities are assumed to be kicks without loss of generality since any Lie map can be factorized into nonlinear kicks up to a desired order [5]. The one-turn map is expressed as

$$
\mathcal{M}=e^{; \tilde{h}_{1}:} e^{: \tilde{h}_{2}:} \ldots e^{: \tilde{h}_{N}:} R_{x, y} R_{\tau}
$$

where $\tilde{h}_{n}$ are the generating functions of the multipolar kicks written in terms of the eigencoordinates $\left(x_{n}, p_{x n}, y_{n}, p_{y n}\right)$ at the longitudinal location $s=s_{n}$. These eigencoordinates are the quantity inside the square brackets of Eq. (8) propagated to the location $s_{n}$ and multiplied by the square root of the beta function at that location. Since Eq. (8) holds at the location just before the ac dipole, the propagation to $s_{n}$ is done by applying $R_{\tau}$ and multiplying by an exponential function with the phase advance at $s_{n}$. The eigencoordinates have the following form:

$$
\begin{aligned}
& x_{n}-i p_{x n}=\sqrt{\beta_{x n}} e^{i \phi_{x n}}\left[\sqrt{2 J_{x}} e^{i\left(\phi_{x}+\phi_{x 0}\right)}+\delta_{x-} e^{i 2 \pi Q_{x D} \tau}-\delta_{x+} e^{-i 2 \pi Q_{x D} \tau}\right], \\
& y_{n}-i p_{y n}=\sqrt{\beta_{y n}} e^{i \phi_{y n}}\left[\sqrt{2 J_{y}} e^{i\left(\phi_{y}+\phi_{y 0}\right)}+\delta_{y-} e^{i 2 \pi Q_{y D} \tau}-\delta_{y+} e^{-i 2 \pi Q_{y D} \tau}\right],
\end{aligned}
$$

where $\beta_{z n}$ and $\phi_{z n}$ are the beta and phase advance functions at the location of the $n$th element. The notation has been generalized to include two independent ac dipoles in the horizontal and vertical planes. The set of eigencoordinates $\left(x_{n}, p_{x n}, y_{n}, p_{y n}\right)$ depends on the initial longitudinal position, which in this case is just before the ac dipole. In Sec. VII a derivation is presented for an arbitrary initial longitudinal position. Using the Campbell-BakerHausdorf theorem, Eq. (9) can be simplified to

$$
\mathcal{M}=e^{: h:} R_{x, y} R_{\tau},
$$

where $h$ can be approximated by

$$
h=\sum_{n=1}^{N} \tilde{h}_{n}+\sum_{n, m<n}^{N}\left[\tilde{h}_{m}, \tilde{h}_{n}\right]+\cdots,
$$

assuming that $\tilde{h}_{n}$ are small enough. The aim of this article is to derive expressions up to first order in the nonlinearities, therefore only the first summation of Eq. (12) will be kept. In the absence of the ac dipole the standard expansion of $h$ is written as

$$
h=\sum_{j k l m} h_{j k l m} z_{x}^{+^{j}} z_{x}^{-^{k}} z_{y}^{+^{l}} z_{y}^{-m}
$$

where $h_{j k l m}$ are the Hamiltonian terms and $z$ are the linearly normalized coordinates defined by

$$
\begin{aligned}
& z_{x}^{ \pm}=\sqrt{2 J_{x}} e^{\mp i\left(\phi_{x}+\phi_{x 0}\right)}, \\
& z_{y}^{ \pm}=\sqrt{2 J_{y}} e^{\mp i\left(\phi_{y}+\phi_{y 0}\right)},
\end{aligned}
$$

where these oscillations would be obtained by applying a kick in either plane. The expansion of $h$ in the presence of the ac dipole is directly computed by replacing the $z$ in Eq. (13) by the quantities inside the brackets of Eq. (10), leading to

$$
h=\sum_{j k l m} h_{j k l m} \xi_{x}^{+^{j}} \xi_{x}^{-^{k}} \xi_{y}^{+^{l}} \xi_{y}^{-m}
$$

with

$$
\begin{aligned}
& \xi_{x}^{ \pm}=\sqrt{2 J_{x}} e^{\mp i\left(\phi_{x}+\phi_{x 0}\right)}+\left|\delta_{x-}\right| e^{\mp i\left(2 \pi Q_{x D} \tau-\eta_{x-}\right)}-\left|\delta_{x+}\right| e^{ \pm i\left(2 \pi Q_{x D} \tau+\eta_{x+}\right)}, \\
& \xi_{y}^{ \pm}=\sqrt{2 J_{y}} e^{\mp i\left(\phi_{y}+\phi_{y 0}\right)}+\left|\delta_{y-}\right| e^{\mp i\left(2 \pi Q_{y D} \tau-\eta_{y-}\right)}-\left|\delta_{y+}\right| e^{ \pm i\left(2 \pi Q_{y D} \tau+\eta_{y+}\right)},
\end{aligned}
$$

where, for convenience, the quantities $\delta_{x, y \pm}$ of Eq. (10) have been separated into an amplitude factor $\left|\delta_{x, y \pm}\right|$ and a phase factor $\exp \left(i \eta_{x, y \pm}\right)$. If the ac dipole is adiabatically turned on the betatron oscillation is not excited; i.e., the terms of Eq. (16) containing $J_{x, y}$ should vanish. Nevertheless, a real ac dipole will always excite the betatron motion up to some level. Therefore the terms containing $J_{x, y}$ are kept until the end of the derivation. 


\section{NORMAL FORM PROCEDURE}

The normal form technique [6,7] can be applied to the one-turn map of Eq. (11) containing Eq. (15). Basically, we look for a new map $e^{: F(J, \phi):}$ which by a similarity transformation brings our one-turn map into a rotation $R_{x, y} R_{\tau} e^{: H(I):}$ that only depends on the new invariants of the motion denoted by $I$. The equation to find the functions $F$ and $H(I)$ is written as

$$
e^{: h:} R_{x, y} R_{\tau}=e^{: F:} e^{: H(I):} R_{x, y} R_{\tau} e^{-: F:} .
$$

This is represented by the following diagram

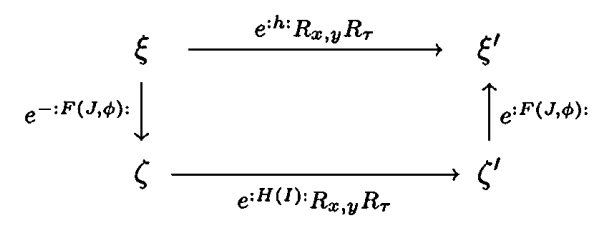

where $\zeta$ represents the normal form coordinates $\left(\zeta_{x}^{+}, \zeta_{x}^{-}\right.$, $\left.\zeta_{y}^{+}, \zeta_{y}^{-}\right)$and $\xi$ represents $\left(\xi_{x}^{+}, \xi_{x}^{-}, \xi_{y}^{+}, \xi_{y}^{-}\right)$. The normal form coordinates are related to the linear coordinates by the expression

$$
\zeta=e^{-: F_{r}: \xi}
$$

where

$$
\begin{aligned}
& \zeta_{x}^{ \pm}=\sqrt{2 I_{x}} e^{\mp i\left(\psi_{x}+\psi_{x 0}\right)}+\left|\delta_{x-}\right| e^{\mp i\left(2 \pi Q_{x D} \tau-\eta_{x-}\right)}-\left|\delta_{x+}\right| e^{ \pm i\left(2 \pi Q_{x D} \tau+\eta_{x+}\right)}, \\
& \zeta_{y}^{ \pm}=\sqrt{2 I_{y}} e^{\mp i\left(\psi_{y}+\psi_{y 0}\right)}+\left|\delta_{y-}\right| e^{\mp i\left(2 \pi Q_{y D} \tau-\eta_{y-}\right)}-\left|\delta_{y+}\right| e^{ \pm i\left(2 \pi Q_{y D} \tau+\eta_{y+}\right)} .
\end{aligned}
$$

$I_{x}$ and $I_{y}$ are the new action invariants, $\psi_{x}$ and $\psi_{y}$ are the new phase variables, and $\tau$ is the same timelike variable as above. By construction the one-turn map of the normal form coordinates is an amplitude dependent rotation represented by

$$
\begin{aligned}
e^{: H(I):} R_{x, y} R_{\tau} \zeta_{x}^{ \pm}= & \sqrt{2 I_{x}} e^{\mp i\left[\psi_{x}+2 \pi Q_{x}(I, \delta)+\psi_{x 0}\right]} \\
& +\left|\delta_{x-}\right| e^{\mp i\left[2 \pi Q_{x D}(\tau+1)-\eta_{x-}\right]}-\left|\delta_{x+}\right| e^{ \pm i\left[2 \pi Q_{x D}(\tau+1)+\eta_{x+}\right]}
\end{aligned}
$$

where $Q_{x}(I, \delta)$ is the betatron tune with its explicit dependence on all the oscillation amplitudes of Eq. (19), $I_{x, y}$ and $\left|\delta_{x, y \pm}\right|$. The formal solutions from Eq. (17) for the functions $F_{r}$ and $H$ up to first order are given by [6]

$$
\begin{gathered}
F_{r}=\frac{1}{1-R_{x, y} R_{\tau}}(h-\bar{h}), \\
H=\bar{h},
\end{gathered}
$$

where $\bar{h}$ represents the average of $h$ over the phase angles, including the new variable $\tau$. In order to obtain a detailed expansion of $F_{r}$ the quantity $(h-\bar{h})$ should be expressed in terms of the eigenvectors of $R_{x, y} R_{\tau}$. These eigenvectors are the summands of the right-hand side (rhs) of Eq. (16) for the initial basis or the summands of the rhs of Eq. (19) for the normal form basis. Nevertheless, in order to deal with simpler expressions this expansion is postponed to the end of the derivation.

\section{THE NONLINEAR MOTION}

Following the same reasoning as in [8] the motion of the particle is computed expanding the exponential operator of Eq. (18) up to first order, resulting in

$$
\boldsymbol{\xi} \approx \boldsymbol{\zeta}+\left[F_{r}, \boldsymbol{\zeta}\right]
$$

where the square brackets denote the Poisson bracket. In order to compute this quantity, the two following properties are used:

$$
\left[\zeta_{x}^{+j}, \zeta_{x}^{-}\right]=-2 i j \zeta_{x}^{+j^{-1}}
$$

$$
\begin{aligned}
& {\left[\frac{1}{1-R_{x, y} R_{\tau}} g(\zeta), \zeta_{x}^{-}\right]} \\
& \quad=\frac{1}{1-e^{-i 2 \pi Q_{x}} R_{x, y} R_{\tau}}\left[g(\zeta), \zeta_{x}^{-}\right],
\end{aligned}
$$

where $g(\zeta)$ is an arbitrary function of the phase space coordinates $\zeta$, which is infinitely differentiable. Therefore the relation of the linearly normalized horizontal coordinate with the normal form horizontal coordinate is

$$
\begin{aligned}
\xi_{x}^{-}= & \zeta_{x}^{-}-2 i \sum_{j k l m} j \frac{h_{j k l m}}{1-e^{-i 2 \pi Q_{x} R_{x, y} R_{\tau}}} \\
& \times \zeta_{x}^{+(j-1)} \zeta_{x}^{-{ }^{k}} \zeta_{y}^{+^{l}} \zeta_{y}^{-^{m}} .
\end{aligned}
$$

As stated above, the eigenvectors of the linear operator $R_{x, y} R_{\tau}$ are the summands of the rhs of Eq. (19) but not the $\zeta$. To obtain the expansion as a power series of the eigenvectors, the $\zeta$ have to be replaced by the expressions of Eq. (19) and the products and powers of the trinomials have to be expanded. The turn-by-turn motion is obtained by successively applying the one-turn map to Eq. (26) as done in Eq. (20).

In the general case, the number of spectral lines arising from Eqs. (26) and (19) in the horizontal motion is large and many Hamiltonian terms contribute to the same spectral line. For instance, the Hamiltonian term $h_{j k 00}$ introduces, in the horizontal spectrum, all the lines with the frequencies expressed as

$$
\left(k_{1}-j_{1}\right) Q_{x}+\left(k_{2}-k_{3}+j_{2}-j_{3}\right) Q_{x D},
$$

$k_{i}$ and $j_{i}$ being arbitrary positive integers or zero fulfilling the following conditions: 


$$
\begin{aligned}
& k_{1}+k_{2}+k_{3}=k, \\
& j_{1}+j_{2}+j_{3}=j-1,
\end{aligned}
$$

where $k$ and $j$ are the indexes of the Hamiltonian term $h_{j k 00}$. Each of the lines given by Eq. (27) has associated the resonance condition expressed as

$$
\begin{array}{r}
\left(k_{1}-j_{1}-1\right) Q_{x}+\left(k_{2}-k_{3}+j_{2}-j_{3}\right) Q_{x D}=p, \\
\text { with } p \in Z .
\end{array}
$$

As an example, using this expression it can be shown that the lowest multipoles driving the resonances $Q_{x D}=$ $p$ and $2 Q_{x D}=p$, with $p \in Z$, are the sextupole and the octupole, respectively. The spectral lines introduced by the Hamiltonian terms $h_{3000}$ and $h_{1020}$ are derived in the Appendix.

Nevertheless, in the ideal case of an adiabatic excitation close enough to the resonance $\left(Q_{D} \approx Q_{x}\right)$, the terms containing either $I_{x, y}$ or $\delta_{x, y+}$ are negligible. Thus Eq. (26), for only one horizontal ac dipole, simplifies to

$$
\xi_{x}^{-} \approx\left|\delta_{x-}\right| e^{i\left(2 \pi Q_{x D} \tau-\eta_{x-}\right)}-\sum_{j k l m} \frac{2 i j h_{j k 00}\left|\delta_{x-}\right|^{(j+k-1)}}{1-e^{i 2 \pi\left[-Q_{x}+(k-j+1) Q_{x D}\right]}} e^{i(k-j+1)\left(2 \pi Q_{x D} \tau-\eta_{x-}\right)} .
$$

In this simplified case the Hamiltonian coefficient $h_{j k 00}$ only introduces the line with frequency $(k-j+1) Q_{x D}$, and the associated resonance condition is

$$
-Q_{x}+(k-j+1) Q_{x D}=p, \quad \text { with } p \in Z .
$$

Although the betatron oscillation has been neglected, the resonance conditions still involve $Q_{x}$. This is due to the factor $e^{-i 2 \pi Q_{x}}$ in the denominator of Eq. (26).

\section{A TRACKING EXAMPLE}

In order to check the expressions derived above, single particle simulations have been done. A ring made of 108 FODO cells has been used, which indeed is a simple model of the Super Proton Synchrotron at CERN. Sextupoles are the only source of nonlinearity. A horizontal ac dipole has been introduced with a linear ramping time of 5000 turns and a flattop of 1000 turns. From Eq. (27) it is concluded that the Hamiltonian terms $h_{3000}, h_{1200}$, and $h_{2100}$ (coming from sextupoles) introduce all lines $(m, n)$ with $|m|+|n| \leq 2$ except the line $(-1,0)$, where $(m, n)$ corresponds to the frequency $m Q_{x}+n Q_{x D}$. In Fig. 1 the Fourier spectrum of the horizontal motion of the

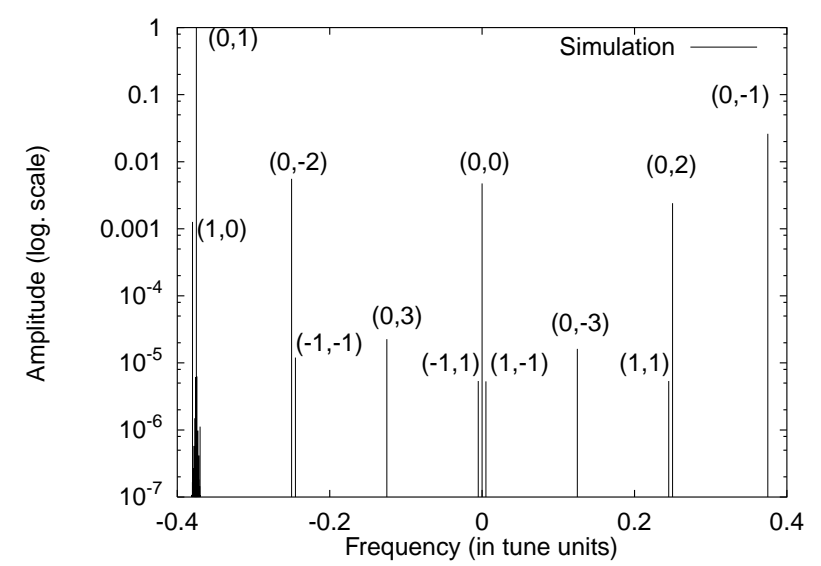

FIG. 1. Normalized Fourier spectrum of the horizontal motion of a particle. The label $(m, n)$ attached to each line indicates that the frequency of that line is $m Q_{x}+n Q_{D}$. For example, the line $(0,2)$ is mainly due to the term $h_{1200}$. simulation is shown. In the figure all the predicted lines are seen plus the $(0,3)$ and $(0,-3)$ lines which come from the second order of the sextupolar terms.

The approximate expression in Eq. (29) gives us the means of unambiguously measuring the Hamiltonian terms from the fast Fourier transform of turn-by-turn data provided the approximation is good enough. The parameters of the simulation were chosen to optimize the study of the third order resonance. The strength of the sextupoles and the Twiss functions at their locations are shown in Table I. In Fig. 2 the prediction of Eq. (29) is compared to the analysis of the simulated tracking by plotting the normalized amplitude of the line $(0,-2)$ versus the

TABLE I. Strength of the sextupoles and Twiss functions at their locations used in the simulation.

\begin{tabular}{cccrcc}
\hline \hline Element & Strength $\left(\mathrm{m}^{-2}\right)$ & $\beta_{x}(\mathrm{~m})$ & $\beta_{y}(\mathrm{~m})$ & $\phi_{x}$ & $\phi_{y}$ \\
\hline Sext. 1 & -0.1 & 42.41 & 55.96 & 6.21 & 6.26 \\
Sext. 2 & -0.2 & 19.63 & 107.64 & 6.53 & 6.53 \\
Sext. 3 & -0.2 & 19.65 & 106.69 & 6.78 & 6.78 \\
\hline \hline
\end{tabular}

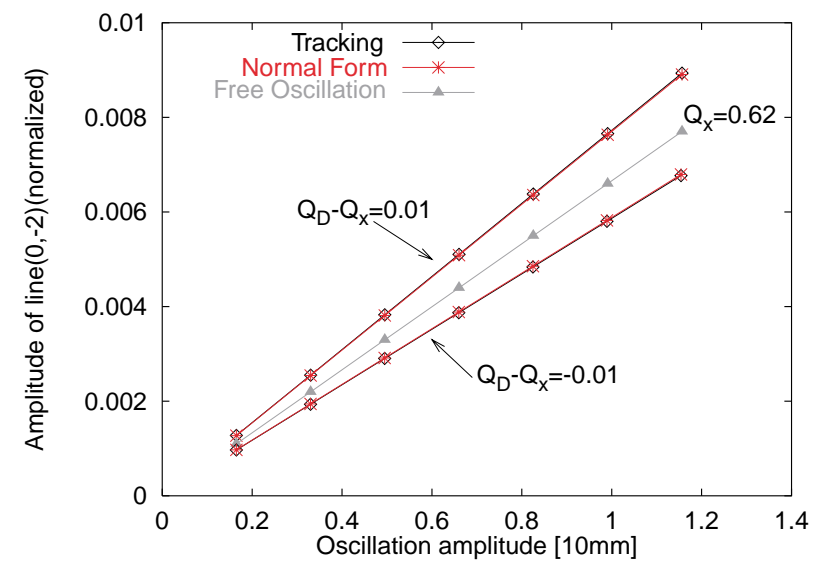

FIG. 2. (Color) Comparison of the normalized amplitude of the line $-2 Q_{D}$ from the horizontal motion of simulated tracking and the approximated model for the two different excitation frequencies $Q_{D}=Q_{x} \pm 0.01$. The normalized amplitude of the line $-2 Q_{x}$ in the free oscillation case is also shown. These lines are due to the resonance term $h_{3000}$. 
oscillation amplitude $\left|\delta_{-}\right|$with $\beta_{x}=100 \mathrm{~m}$. The comparison is done for two excitation frequencies. The limit of the normalized amplitude of the line $(0,-2)$ as it approaches the resonance $Q_{D}=Q_{x}$ is also shown in the picture. In this limit the motion is equivalent to a free oscillation produced by a single kick. The normalized amplitude of the line $(0,-2)$ with $Q_{D}=Q_{x}$ equals the normalized amplitude of the line with frequency $-2 Q_{x}$ from the kicked particle motion.

\section{CONSIDERING AN ARBITRARY INITIAL LOCATION}

As stated in Sec. III, the eigenvectors of Eq. (10) depend on the initial location, i.e., the longitudinal coordinate with zero phase advance. The turn-by-turn motion at an arbitrary location has been calculated in Eq. (5), rewriting it using the one-turn linear operators yields

$$
\hat{x}(T)-i \hat{p}_{x}(T)=\left(R_{x} \otimes R_{\tau}\right)^{T}\left[\sqrt{2 J} e^{i\left(\phi_{x}+\phi_{x 0}\right)}+e^{-i \phi_{x D}}\left(\delta_{-} e^{i 2 \pi Q_{D} \tau}-\delta_{+} e^{-i 2 \pi Q_{D} \tau}\right)\right] .
$$

The eigencoordinates $\left(x_{n}, p_{x n}, y_{n}, p_{y n}\right)$ at the $n$th element will be given by different expressions depending on whether it is located before or after the ac dipole. To compute them the expression inside the square brackets of Eq. (31) is propagated to $s_{n}$ as done in Sec. III, but now $R_{\tau}$ is applied only when $\phi_{x n}>\phi_{x D}$. This is given by the following expressions:

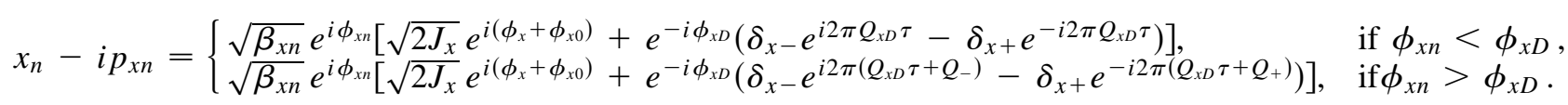

Similar expressions hold for the vertical coordinate. Following the steps of Sec. III, the Hamiltonian $h$ is expressed up to first order in $\tilde{h}_{n}$ as

$$
h=\sum_{n=1}^{N} \tilde{h}_{n}\left(x_{n}, p_{x n}, y_{n}, p_{y n}\right) .
$$

It was possible [see Eq. (15)] to express the Hamiltonian with an ac dipole using the Hamiltonian terms $h_{j k l m}$ because all the elements fell into one of the two possible solutions of Eq. (32). In the general case of an arbitrary initial location, the contribution of each element to $h$ depends on its relative position with respect to the ac dipole. To achieve a similar expansion to Eq. (15), the Hamiltonian in the absence of ac dipole is separated into two summations in the following way:

$$
\begin{aligned}
h & =\sum_{\phi_{x n}<\phi_{x D}} \tilde{h}_{n}+\sum_{\phi_{x n}>\phi_{x D}} \tilde{h}_{n} \\
& =\sum_{j k l m}\left(h_{j k l m}^{<}+h_{j k l m}^{>}\right) z_{x}^{+^{j}} z_{x}^{-^{k}} z_{y}^{+^{l}} z_{y}^{-^{m}},
\end{aligned}
$$

where $h_{j k l m}^{<}$contains the contributions of all the elements before the ac dipole and $h_{j k l m}^{>}$the elements after. These new terms can be used in expanding the Hamiltonian containing an ac dipole, leading to the expression

$$
\begin{aligned}
h= & \sum_{j k l m} h_{j k l m}^{<} \xi_{<x}^{+j} \xi_{<x}^{-^{k}} \xi_{<y}^{+^{l}} \xi_{<y}^{-m} \\
& +\sum_{j k l m} h_{j k l m}^{>} \xi_{>x}^{+j} \xi_{>x}^{-^{k}} \xi_{>y}^{+^{l}} \xi_{>y}^{-m},
\end{aligned}
$$

with

$$
\begin{aligned}
& \xi_{<x}^{ \pm}=\sqrt{2 J_{x}} e^{\mp i\left(\phi_{x}+\phi_{x 0}\right)}+\left|\delta_{x-}\right| e^{\mp i\left(2 \pi Q_{x D} \tau+\eta_{x-}^{\prime}\right)}-\left|\delta_{x+}\right| e^{ \pm i\left(2 \pi Q_{x D} \tau+\eta_{x+}^{\prime}\right)}, \\
& \xi_{>x}^{ \pm}=\sqrt{2 J_{x}} e^{\mp i\left(\phi_{x}+\phi_{x 0}\right)}+\left|\delta_{x-}\right| e^{\mp i\left[2 \pi\left(Q_{x D} \tau+Q_{-}\right)+\eta_{x-}^{\prime}\right]}-\left|\delta_{x+}\right| e^{ \pm i\left[2 \pi\left(Q_{x D} \tau+Q_{+}\right)+\eta_{x+}^{\prime}\right]},
\end{aligned}
$$

and $\eta_{x \pm}^{\prime}=\mp\left(\eta_{x \pm}-\phi_{x D}\right)$. Similar expressions hold for the vertical coordinates. The turn-by-turn motion can be computed in the same way as was done in Sec. V obtaining

$$
\xi_{x}^{-}=\zeta_{x}^{-}-2 i \sum_{j k l m} j \frac{1}{1-e^{-i 2 \pi Q_{x}} R_{x, y} R_{\tau}}\left(h_{j k l m}^{<} \zeta_{<x}^{+(j-1)} \zeta_{<x}^{-k} \zeta_{<y}^{+l} \zeta_{<y}^{-m}+h_{j k l m}^{>} \zeta_{>x}^{+(j-1)} \zeta_{>x}^{-k} \zeta_{>y}^{+l} \zeta_{>y}^{-m}\right),
$$

where the coordinates $\zeta$ are defined as the coordinates $\xi$ in Eq. (36) but replacing $J_{x}$ by $I_{x}$ and $\phi_{x}$ by $\psi_{x}$. Again the same approximation can be done as in Sec. V, resulting in the following expression for the horizontal motion in presence of one horizontal ac dipole:

$$
\xi_{x}^{-} \approx\left|\delta_{x-}\right| e^{i\left(2 \pi Q_{x D} \tau+\eta_{x-}^{\prime}\right)}-2 i \sum_{j k l m} j \frac{h_{j k 00}^{\prime}\left|\delta_{x-}\right|^{(j+k-1)}}{1-e^{i 2 \pi\left[-Q_{x}+(k-j+1) Q_{x D}\right]}} e^{i(k-j+1)\left(2 \pi Q_{x D} \tau+\eta_{x-}^{\prime}\right)},
$$

with

$$
h_{j k 00}^{\prime}=h_{j k 00}^{<}+h_{j k 00}^{>} e^{i(k-j+1) 2 \pi Q_{-}}
$$




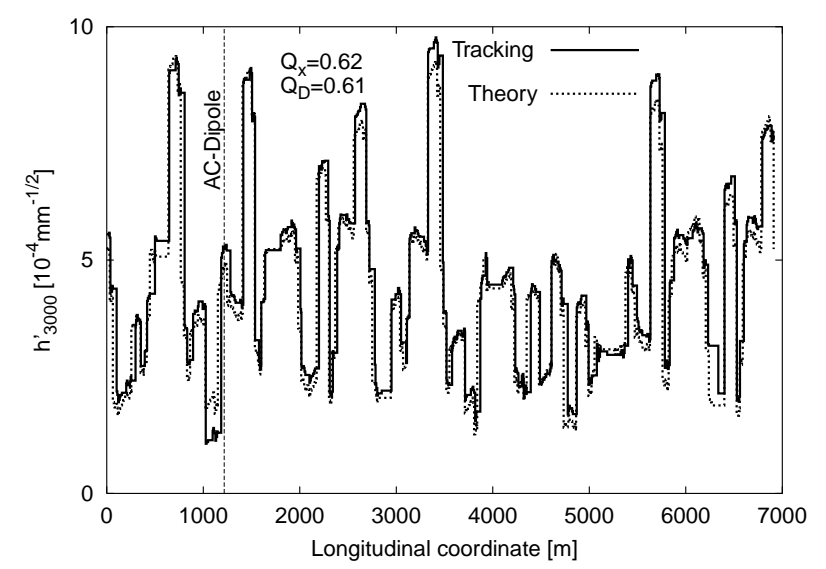

FIG. 3. Comparison of the amplitude of the Hamiltonian term $h_{3000}^{\prime}$ computed from tracking and from theory (both with ac dipole) plotted versus the longitudinal position.

This expression shows the difference between the Hamiltonian terms of a lattice with and without an ac dipole. For those locations where all the sources are seen to be either before $\left(h^{<}\right)$or after $\left(h^{>}\right)$the ac dipole, the amplitudes of the two kinds of terms, $h^{\prime}$ and $h$, are equal. Notice that as $Q_{-}$decreases the terms with an ac dipole tend to the terms without an ac dipole. Also notice that the high order terms, with larger $|k-j+1|$, will exhibit greater discrepancies between the two types of Hamiltonian terms. At the longitudinal location of a magnetic source the corresponding Hamiltonian terms vary abruptly in phase and amplitude [9]. The amplitude remains constant between sources. This property will allow the identification of locations with multipolar kicks in a real machine by using beam position data from the pickups along the ring.

A tracking simulation has been performed to check the approximation used in deriving Eq. (38). The same lattice and method of the previous tracking example in Sec. VI



FIG. 4. Comparison between the theoretical amplitudes of the Hamiltonian terms $h_{3000}^{\prime}$ (with ac dipole) and $h_{3000}$ (free oscillation) plotted versus the longitudinal position. have been used, but 108 sextupoles were introduced. Turn-by-turn data are obtained at the different longitudinal locations. The Hamiltonian term $h_{3000}^{\prime}$ is obtained by evaluating the amplitude of the $(0,-2)$ spectral line of these data [according to Eq. (38)]. The results of this analysis together with the theoretical value of $h_{3000}^{\prime}$ obtained from the lattice parameters are shown in Fig. 3. Small discrepancies arise at some locations due to contributions from the terms $h_{1200}$ and $h_{2100}$ to the spectral line $(0,-2)$. These contributions are proportional to the quantity $\delta_{x+}$ and they were neglected to arrive at Eq. (38). To give an idea of the differences between the Hamiltonian terms with and without ac dipole their amplitudes are plotted versus the longitudinal coordinate for the same lattice in Fig. 4.

\section{CONCLUSION}

A method to derive the normal form of a Hamiltonian with an ac dipole term is presented. To avoid the explicit time dependence of the Hamiltonian a new dimension is introduced. In principle this method works not only for the case of an ac dipole but for all cases where there is a solution of the linear plus the time-dependent parts of the Hamiltonian.

The turn-by-turn motion at any location of the lattice is derived using Lie algebra techniques. In the general case, various Hamiltonian terms contribute to the same Fourier spectral line of the motion. This is not the desired situation when using the ac dipole for nonlinear beam diagnostic. Nevertheless, an approximate expression for the turn-by-turn motion is derived under the ideal conditions that the excitation is adiabatic and the ac dipole tune is sufficiently close to the natural tune. It has been shown that the Hamiltonian terms in the presence of the ac dipole are different from those without ac dipole. Nevertheless, the discrepancies should not be large and the local information contained in both cases is equivalent; i.e., it is possible to identify longitudinal locations with multipolar kicks. All these predictions have been compared with tracking simulations in a FODO lattice with sextupoles. In the more academic example, where one resonance is strongly excited, the agreement was excellent. In the more general case, where 108 sextupoles were introduced, some small discrepancies arose due to contributions from different resonance terms to the same spectral line.

\section{ACKNOWLEDGMENTS}

The author thanks Dr. Frank Schmidt for the supervision of this work and for his help in modifying the tracking code SIXTRACK to include an ac dipole element. I am also thankful to Dr. O. Brüning, Dr. M. Hayes, Dr. S. Peggs, and Dr. F. Ruggiero for reading the manuscript and making valuable comments. 


\section{APPENDIX: SPECTRAL LINES INTRODUCED BY} $\mathrm{H}_{3000}$ AND $\mathrm{H}_{1020}$

From Eq. (26) the term $h_{3000}$ (which at the ac dipole is equal to the term $h_{3000}^{\prime}$ ) contributes to the horizontal motion with the quantity

$$
\frac{-6 i h_{3000}}{1-e^{-i 2 \pi Q_{x}} R_{x, y} R_{\tau}} \zeta_{x}^{+^{2}}
$$

where $\zeta^{+x}$ is given by

$$
\begin{aligned}
\zeta_{x}^{+}= & \sqrt{2 I_{x}} e^{-i\left(\psi_{x}+\psi_{x 0}\right)}+\left|\delta_{x-}\right| e^{-i\left(2 \pi Q_{x D} \tau-\eta_{x-}\right)} \\
& -\left|\delta_{x+}\right| e^{+i\left(2 \pi Q_{x D} \tau+\eta_{x+}\right)} .
\end{aligned}
$$

$$
\begin{aligned}
\zeta_{x}^{+^{2}}= & 2 I_{x} e^{-i 2\left(\psi_{x}+\psi_{x 0}\right)}+\left|\delta_{x-}\right|^{2} e^{-i 2\left(2 \pi Q_{x D} \tau-\eta_{x-}\right)}+\left|\delta_{x+}\right|^{2} e^{+i 2\left(2 \pi Q_{x D} \tau+\eta_{x+}\right)}+2 \sqrt{2 I_{x}}\left|\delta_{x-}\right| e^{-i\left(\psi_{x}+\psi_{x 0}\right)-i\left(2 \pi Q_{x D} \tau-\eta_{x-}\right)} \\
& -2 \sqrt{2 I_{x}}\left|\delta_{x+}\right| e^{-i\left(\psi_{x}+\psi_{x 0}\right)+i\left(2 \pi Q_{x D} \tau+\eta_{x+}\right)}-2\left|\delta_{x-}\right|\left|\delta_{x+}\right|
\end{aligned}
$$

The frequencies of the lines introduced by this expression are $-2 Q_{x},-2 Q_{x D}, 2 Q_{x D},-Q_{x}-Q_{x D},-Q_{x}+Q_{x D}$, and 0 .

By virtue of Eq. (A1), the resonance conditions corresponding to the different lines are $-3 Q_{x}=p, \quad-2 Q_{x D}-Q_{x}=p, \quad 2 Q_{x D}-Q_{x}=p$, $-2 Q_{x}-Q_{x D}=p,-2 Q_{x}+Q_{x D}=p$, and $-Q_{x}=p$, with $p \in Z$.

The equivalent calculation will be done now for the term $h_{1020}$. From Eq. (26) the term $h_{1020}$ contributes to the horizontal motion with the quantity

$$
\frac{-2 i h_{1020}}{1-e^{-i 2 \pi Q_{x}} R_{x, y} R_{\tau}} \zeta_{y}^{+^{2}}
$$

where $\zeta_{y}^{+}$is given by

$$
\begin{aligned}
\zeta_{x}^{+} & =\sqrt{2 I_{y}} e^{-i\left(\psi_{x}+\psi_{y 0}\right)}+\left|\delta_{y-}\right| e^{-i\left(2 \pi Q_{y D} \tau-\eta_{y-}\right)} \\
& -\left|\delta_{y+}\right| e^{+i\left(2 \pi Q_{y D} \tau+\eta_{y+}\right)}
\end{aligned}
$$

Expanding the square $\zeta_{y}^{+^{2}}$ in Eq. (A4) gives

$$
\begin{aligned}
\zeta_{y}^{+^{2}}= & 2 I_{y} e^{-i 2\left(\psi_{y}+\psi_{y 0}\right)}+\left|\delta_{y-}\right|^{2} e^{-i 2\left(2 \pi Q_{y D} \tau-\eta_{y-}\right)}+\left|\delta_{y+}\right|^{2} e^{+i 2\left(2 \pi Q_{y D} \tau+\eta_{y+}\right)}+2 \sqrt{2 I_{y}}\left|\delta_{y-}\right| e^{-i\left(\psi_{x}+\psi_{y 0}\right)-i\left(2 \pi Q_{y D} \tau-\eta_{y-}\right)} \\
& -2 \sqrt{2 I_{y}}\left|\delta_{y+}\right| e^{-i\left(\psi_{y}+\psi_{y 0}\right)+i\left(2 \pi Q_{y D} \tau+\eta_{y+}\right)}-2\left|\delta_{y-}\right|\left|\delta_{y+}\right|
\end{aligned}
$$

The frequencies of the lines introduced by this expression are $-2 Q_{y},-2 Q_{y D}, 2 Q_{y D},-Q_{y}-Q_{y D},-Q_{y}+Q_{y D}$, and 0 .

By virtue of Eq. (A4), the resonance conditions corresponding to the different lines are $-2 Q_{y}-Q_{x}=p,-2 Q_{y D}-Q_{x}=p, 2 Q_{y D}-Q_{x}=$ $p,-Q_{y}-Q_{x}-Q_{x D}=p,-Q_{y}-Q_{x}+Q_{x D}=p$, and $-Q_{x}=p$, with $p \in Z$.

[1] I. Borchardt, E. Karantzoulis, H. Mais, and G. Ripken, DESY Report No. DESY-87-161, 1987.

[2] S. Peggs and C. Tang, Brookhaven National Laboratory Report No. RHIC/AP/159, 1998.
[3] H. Goldstein, Classical Mechanics (Addison-Wesley, Reading, MA, 1980), p. 368.

[4] É. Forest, J. Math. Phys. (N.Y.) 31, 1133-1144 (1990).

[5] J. Irwin, Superconducting Super Collider Laboratory Report No. SSC-228, 1989.

[6] M. Berz, É. Forest, and J. Irwin, Part. Accel. 24, 91-107 (1989).

[7] A. Bazzani, E. Todesco, G. Turchetti, and G. Servizi, CERN Report No. CERN 94-02, 1994.

[8] R. Bartolini and F. Schmidt, Part. Accel. 59, 93-106 (1998).

[9] F. Schmidt, R. Tomás, and A. Faus-Golfe, in Proceedings of the Particle Accelerator Conference, Chicago, 2001, edited by P. Lucas and S. Webber (IEEE, Piscataway, NJ, 2001). 